\title{
Workshop Docente de Estructuras Desplegables Utilizando Materiales Básicos
}

\author{
José Miguel Molines Cano ${ }^{1}$, Igor Fernández Plazaola ${ }^{2}$ Jaime Llinares Millán ${ }^{3}$ \\ Maria Eugenia Torner Feltrer ${ }^{1}$, María Luisa Navarro García ${ }^{3}$ y Francisco Sanchis \\ Sampedro4. \\ 1 Universitat Politècnica de Valencia. Dpto mecánica medios continuos y TE, \\ jomoca1@upv.es; marieutf@hotmail.com
}

${ }^{2}$ Universitat Politècnica de Valencia. Dpto organización de empresas, iplazaola@doe.upv.es

3 Universitat Politècnica de Valencia. Dpto construcciones arquitectónica, jllinares@csa.upv.es; mlnavar@csa.upv.es.

${ }^{4}$ Barreira Escuela de Diseño. Jefe de Dpto de Diseño de Interiores

Sanchis.p@barreira.edu.es

\begin{abstract}
Included within the teaching framework proposed by the School of Engineering Building, within the day of the EXCO, the workshop "drop-down structures using basic materials" is incorporated.

The main objective of the workshop is to incorporate a new educational methodology within the scope of the structures. Mainly it is intended that a group of students can build with their own hands a singular structure with design features proposed for them.

For the construction of this appliance, students work with conventional materials and supply for all, so that they understand the functionality of the mock as a scale model capable of representing the reality of a structure designed a priori and the potential of this system practical in the design of structural element.

In this way is intended for students at the end of the workshop fully understand the operation of these tipology and can replace the preconceived theoretical idea of the way we teach the behavior of structures.
\end{abstract}

Keywords: drop-down structure, structural desing, scale models, workshop.

\section{Resumen}

Incluido dentro del marco docente propuesto por la Escuela Técnica Superior de Ingenieria de Edificación, dentro de la jornada de la EXCO, se incorpora el taller "Estructuras despleglables utilizando materiales básicos".

El principal objetivo del taller consiste en incorporar una nueva metodología educativa dentro del ámbito de las estructuras. Principalmente se pretende 
que un grupo de alumnos construyan con sus propias manos una estructura singular con unas características de diseño propuestas por ellos.

Para la construcción de este artefacto, los alumnos trabajaran con materiales convencionales y al alcance de todos, de forma que comprendan la funcionalidad de la maqueta como un modelo a escala capaz de representar la realidad de una estructura diseñada a priori y el potencial de este sistema práctico en la concepción de elemento estructural.

Con ello, se pretende que el alumno al finalizar el taller entienda perfectamente el funcionamiento de estas tipologías y sustituya la concepción teórica preconcebida de la forma de enseñar el comportamiento de las estructuras.

Palabras clave: estructuras desplegables, cálculo estructural, modelo a escala, workshop.

\section{Introducción}

Las metodologías didácticas son uno de los componentes fundamentales de los proyectos formativos (Fernández, 2003). Estas metodologías han ido evolucionando y avanzando con el paso del tiempo. De entre las nuevas metodologías, una de las más importantes por las que abogan las nuevas políticas educativas es el aprendizaje a través de la práctica. Se centra en una enseñanza individualizada del alumno a través de ejemplos claros o un problema planteado a priori, lo que provoca que el alumno investigue y avance en sus conocimientos a través de su propia experiencia (Heyman, 1999).

Dentro del campo de la ingeniería, el conocimiento estructural es una de las principales vertientes de enseñanza, que en ocasiones resulta tediosa e incomprensible por una gran muestra del alumnado. Parte de este fracaso se centra en la desmotivación que presentan los alumnos frente a una enseñanza normalmente teórica, incompresible y lejana a la realidad que les rodea. Sin embargo y aunque parece lógico que las nuevas metodologías docentes aboguen más por una enseñanza dinámica en la que el alumno se convierte en el protagonista, no se puede perder de vista una previa base teórica capaz de dar los conocimientos previos para desarrollo individualizado y práctico que se pretende.

Por ello y dentro del marco educativo que ofrece cada año la Escuela Técnica Superior de Ingeniería de la Edificación en sus jornadas EXCO (Exposición de Construcción dentro de la feria internacional CEVISAMA), se propone un taller titulado "Construcción de Estructuras Desplegables Utilizando Materiales Básicos" que engloba de forma generalizada conceptos desarrollados en diversas disciplinas existentes en la mayoría de las ingenierías que se imparten en la UPV.

Este trabajo, trata de explicar los objetivos, metodología y conclusiones extraídas del taller, centrado básicamente en una metodología eminentemente práctica. Sin embargo, y aunque es incomprensible el desarrollo del taller sin una base teórica previa del estudiante, lo que

(cc) EY-NC-ND 2016, Universitat Politècnica de València 
pretende es evitar largas exposiciones teóricas sustituyéndolas por ejemplos de aplicación práctica.

\section{Objetivos}

- Dotar al alumno de visión analítica de las estructuras a través de herramientas sencillas a escala. La experiencia que puede adquirir el alumno puede serle de gran ayuda para aplicarla en distintas fases de su formación, incluso en su vida laboral futura. Este proceso le permite comprobar de forma instantánea el diseño de cualquier estructura pensada e imaginada por el alumno , además de ofrecerle una coherencia formal en cuanto al factor a escala a emplear.

- Ofrecer al alumno una nueva herramienta práctica capaz de suplir la concepción teórica que tenían de los métodos teóricos estructurales.

- Mostrar el potencial de los materiales no convencionales o reciclados como elementos perfectamente útiles para el desarrollo de maquetas. Estos materiales perfectamente capacitados, pueden serles de gran ayuda para la comprobación y realización de maquetas, pero a su vez les permite comprobar la versatilidad de comportamiento que tiene cada material de forma real, relacionándolos con los datos experimentales de otros materiales que conocen y tienen asumidos como habituales de su formación docente.

- Explicar y recordar algunos conceptos estructurales mediante la creación del modelo.

- Presentar al alumno el diseño de estructuras diferentes a las soluciones conocidas y habituales. Se pretende que el alumno comprenda que las estructuras pueden ir mucho más allá de la concepción que tienen preestablecida y que el avance y estudio que se realiza sobre ella abre nuevas posibilidades ingenieriles y arquitectónicas que desconoce.

\section{Sobre las Estructuras Despleglables}

Cuando se habla de estructuras desplegables es imprescindible hablar de Emilio Pérez Piñero, pionero en su estudio desde los años 60' y su discípulo Félix Escrig Pallarés años después. Piñero fue el primer investigador que mostró soluciones espaciales para este tipo de estructuras, con propuestas que incluso hoy en día han sido incomprendidas por investigadores posteriores (Puertas, 1989). Sin embargo y dada la versatilidad que ofrece este tipo de estructuras, resulta interesante ser objeto de estudio.

Emilio Pérez Piñero, a diferencia de otros investigadores, no parte de modelos teóricos para la concepción y el diseño de este tipo de estructuras, si no todo lo contrario, trabaja con ellas a partir de pequeñas maquetas a escala. Estos elementos a escala parten de su mente como elementos totalmente innovadores, sin una base anterior en la que apoyarse. Por ello, la habilidad manual y la visión espacial desarrollada por Piñero ha sido la base fundamental que ha despertado gran interés a numerosos investigadores tanto a nivel nacional como mundial desde mitad del siglo pasado.

(c)) EY-NC-ND 2016, Universitat Politècnica de València

Congreso In-Red (2016) 

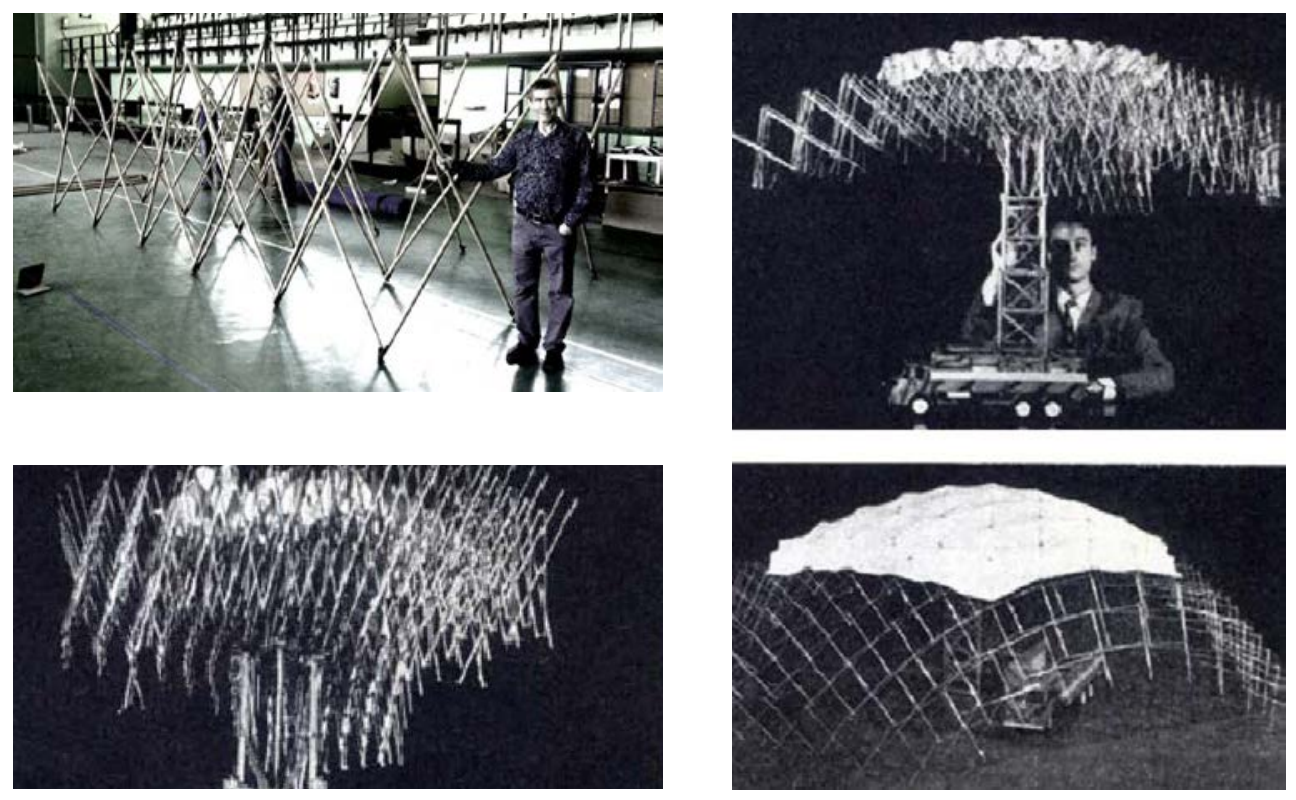

Fig. 1 Modelos estructurales desarrollados por Piñero

Años después, su discípulo Escrig Pallarés desarrolla en base a los diseños de Piñero las estructuras desplegables basadas en el mecanismo tijera. El mecanismo de tijera es el básico para permitir el despliegue, que según el punto de articulación de las barras y la forma de éstas, se pueden generar estructuras de superficie curva.

Para comprender el potencial de estos sistemas, basta con entender que una superficie no puede sostenerse por sí sola. Para ello debe tener una estructura normalmente geométrica asociada que lo convierta en un elemento portante.

Dada la naturaleza de estas estructuras, y sobre todo de su carácter móvil, para su concepción, es necesario pensar tanto geométricamente como matemáticamente para poder diseñar de forma exacta un elemento estable y comprender su funcionamiento. (Sánchez, 1996).

Sin embargo, la complejidad geométrica que supone la representación gráfica de este tipo de estructuras, hace impensable un estudio de otra forma diferente a un análisis práctico mediante maquetas, tal y como inició Piñero y siguieron sus sucesores. 

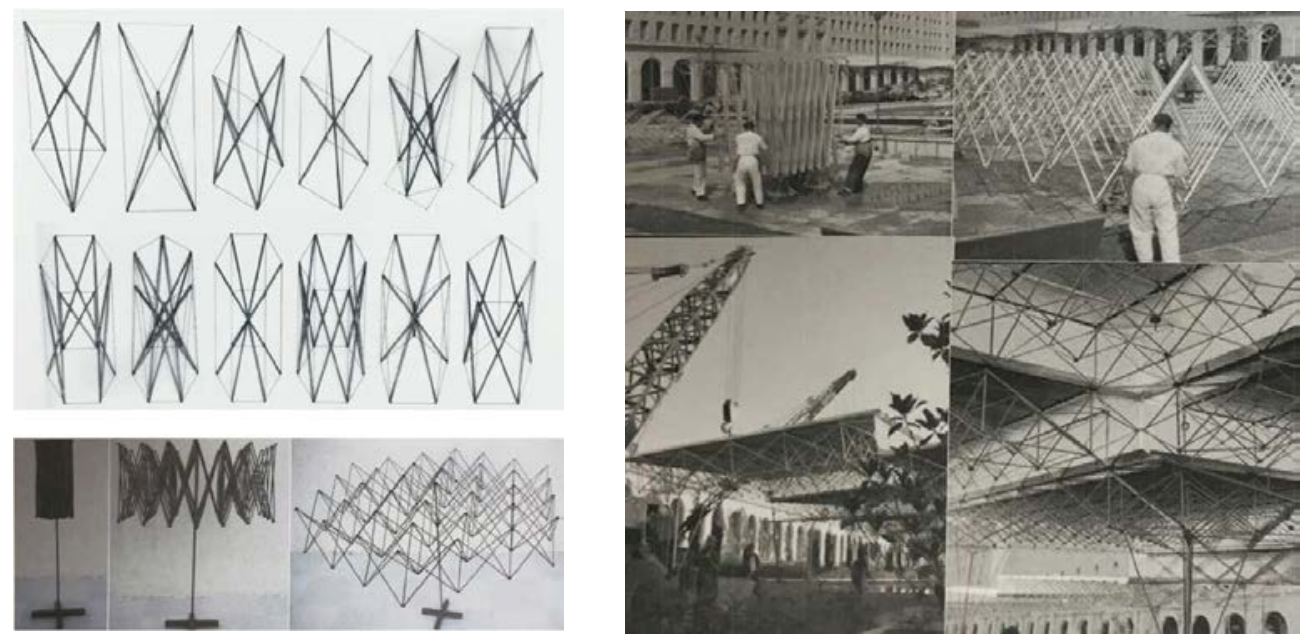

Fig. 2 Prototipos desarrollados por Piñero y Escrig de estructuras plegables.

En consecuencia el taller que se propone pretende desarrollar una aptitud analítica de las estructuras mediante la ejecución de una estructura desplegable. Dentro de él se pretende que el alumno desarrolle una concepción geométrica y matemática de las estructuras, y en base a ello la capacidad de diseñar una estructura, en este caso desplegable, acorde a una necesidad real y concreta.

\section{Importancia de la maqueta en la concepción estructural}

De forma semejante a los bocetos, las maquetas tienen un papel fundamental en la fase del diseño arquitectónico y estructural. Más aún cuando se habla de estructuras singulares como las que se pretende desarrollar en el taller, difíciles de representar gráficamente.

Se pueden distinguir dos tipos de maquetas, las de representación final y la correspondiente al estudio. Las primeras de ellas tienen una vinculación meramente comercial y las segundas dan sentido y acompañan al proceso de diseño y concepción de una idea del ingeniero o arquitecto. Estas maquetas de estudio, han sido de gran valor en siglos anteriores como elemento fundamental en la representación a escala de cualquier elemento que se quería construir. (Torroja, 1960). Uno de los precursores más conocidos es Gaudí. Sus maquetas de trabajo son famosas por la utilidad y ayuda que representan en la construcción de edificio singulares como la cripta Güell o la Sagrada Familia.

"Arquitectos como Félix Candela, Frei Otto, Pier Luigi Nervi... e ingenieros como Eduardo Torrja, Heinz Isler... comenzaron a realizar ensayos científicos sobre modelos físicos reducidos, que aunque con un carácter diferente, buscaban cuantificar el uso de las maquetas en búsqueda de la geometría más eficaz desde el punto de vista estructural" (Cassinello 2006)

Sin embargo el auge progresivo del mundo digital ha desvirtuado los modelos tangibles que ofrecían las maquetas, mostrándolos como elementos obsoletos sin posibilidades y poco 
competitivos. No obstante la simulación virtual de las diferentes componentes que envuelven cualquier propuesta, aunque ofrece unas posibilidades que dejan fuera de competición a la maqueta tradicional, no es comparable a las posibilidades prácticas, educativas y tangibles que ofrecen los métodos tradicionales.

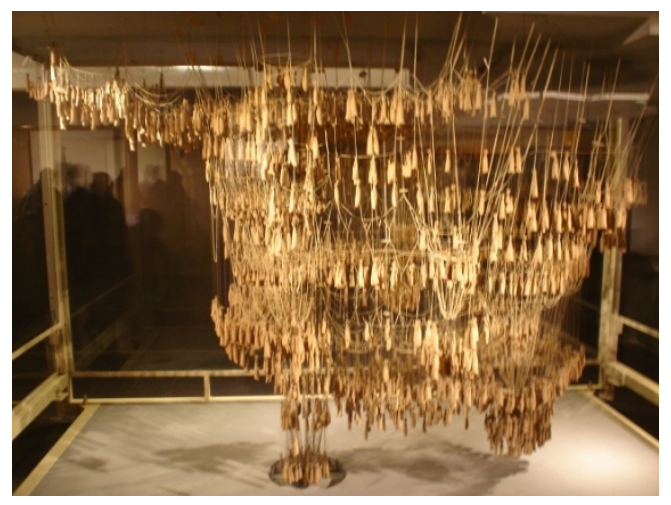

Fig. 3 Funicular utilizado por Gaudí para calcular la Sagrada Familia

En consecuencia, y dado el potencial que ofrecen las maquetas como elemento educativo y de estudio, este taller pretende mostrar la maqueta como un elemento útil y de gran ayuda para el análisis y comprensión de estructuras. Además pretende afianzar los conocimientos adquiridos por el estudiante, ampliarlos y ofrecer una nueva metodología de trabajo en el campo estructural.

\section{Desarrollo de la Innovación}

El taller que se presenta, tal y como se introduce en el primer punto de este articulo, se desarrolla dentro del ámbito de la Feria de la Tecnología de la Construcción EXCO 2016, con una duración de 5 horas. Al igual en años anteriores, desde la Escuela Técnica Superior de Ingeniería de Edificación se apuesta por la organización de talleres dirigidos a estudiantes de la UPV, centrados en nuevas metodologías docentes enfocadas al espíritu del nuevo espacio europeo de educación superior.

Para ello y con el apoyo de la organización de la EXCO '16, puso a disposición de los talleres los medios necesarios para el desarrollo de esta innovación docente. En el ámbito del taller que se propone, se requería de un espacio suficientemente grande para poder realizar una maqueta de unas dimensiones considerables y espacio para trabajo manual del material, provistos del utillaje de montaje necesarios.

Una vez comprobado que todo el montaje era correcto se inicia el taller con la participación de 12 alumnos. Debido a la complejidad que presentan este tipo de estructuras, para muchos de ellos totalmente desconocidas, se prepara un dossier previo en previsión de iniciar el taller con una pequeña explicación teórica.

Desde el inicio, se pretende un enfoque inminentemente práctico, con lo que la explicación teórica que se ofrece al alumno se centra básicamente en la proposición de ejemplos de estructuras conocidas, muchas de ellas en la propia universidad y alrededores, precursoras de 
lo que se conoce actualmente como estructuras desplegables. Los únicos conceptos necesarios que se proponen son el esfuerzo a compresión y tracción, fácilmente comprensible por todos. Además se expone la importancia que supuso el uso del acero como nuevo material dentro del campo estructural, el avance de los nuevos modelos que trajo consigo y el desarrollo que han sufrido este tipo de armazones.

El siguiente punto a desarrollar son las uniones. Debido al poco tiempo del que se disponía, se plantearon a priori diversas posibilidades sencillas que cumpliesen con las expectativas que se pretendían. Para ello se muestra un prototipo construido con anterioridad al taller de forma que los alumnos comprendan el sistema y sean capaces de reproducirlo a continuación.
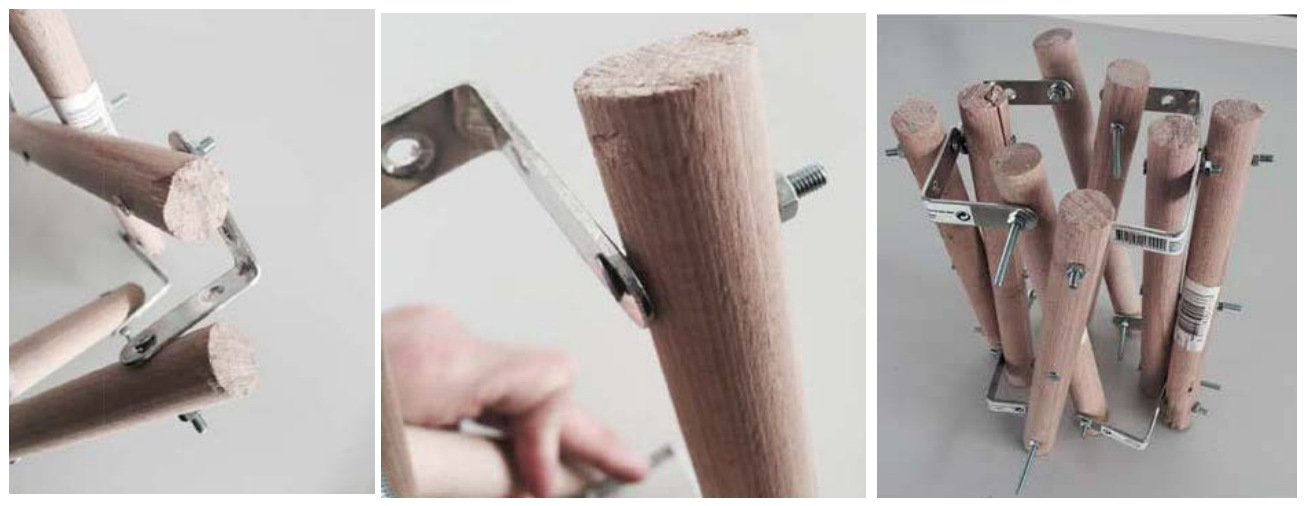

Fig. 4 Muestra el montaje y estado final de un módulo de la estructura desplegable.

Sin embargo en este caso, el material a emplear será sensiblemente distinto. Se pretende el uso de materiales convencionales, comúnmente conocidos por los alumnos, como son palos de escoba, tornillos y unas escuadras como las del prototipo anterior.

Llegados a este punto, los alumnos son capaces de confeccionar con sus propias manos una estructura desplegable, semejante a las que se han planteado como ejemplo inicialmente. Y con todo ello, el alumno asume una responsabilidad semejante a la que toma el ingeniero o arquitecto durante el diseño y concepción de una estructura real.

Para el montaje de los distintos módulos que confeccionarán el artefacto, el trabajo se divide en dos fases. En la primera se realizarán las operaciones de taladro y corte de barras (palos de escoba); y la segunda las operaciones de ensamblaje y ajuste final.
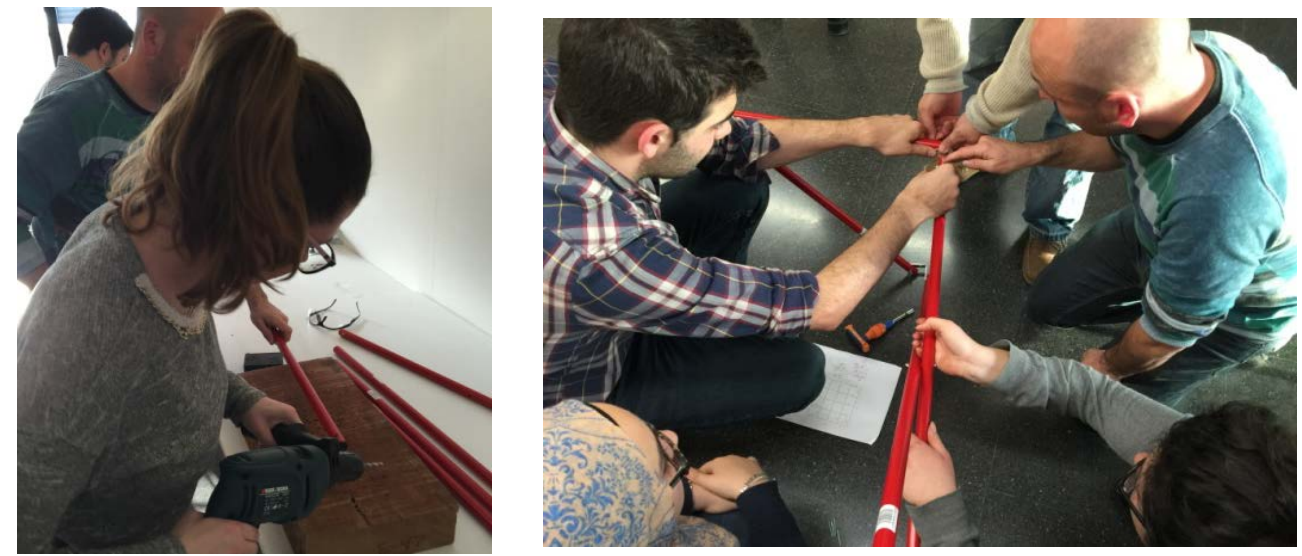

(c)) EY-NC-ND 2016, Universitat Politècnica de València

Congreso In-Red (2016) 
Fig. 5 Preparación de los módulos y proceso de ensamblaje.

Por último, tras el montaje de la estructura propuesta diseñada por los propios alumnos siguiendo uno de los ejemplos propuestos, el siguiente estadio supone la comprobación de su capacidad sustentante y su carácter desmontable, que implica el plegado y desplegado sencillo, además de su estabilidad aparente al menos de su propio peso.
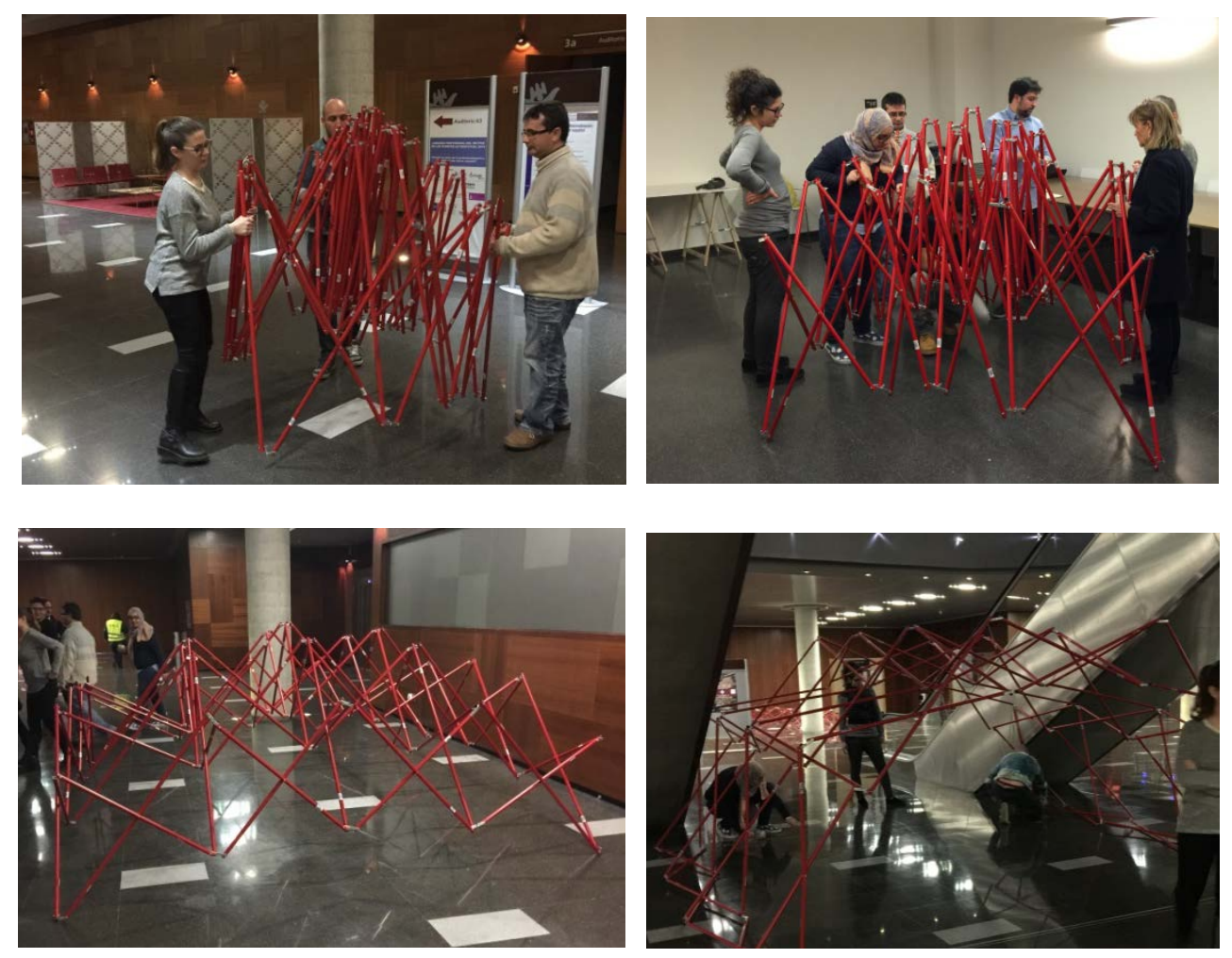

Fig. 6 Comprobación final de la estructura desmontable. Modelo expuesto en la EXCO '15.

\section{Resultados}

Tras finalizar el taller se pueden analizar los distintos resultados obtenidos:

1. La aplicación de las metodologías activas de enseñanza-aprendizaje en el campo de las estructuras. Los resultados obtenidos superaron las expectativas del taller, puesto que el alumno se mostraba motivado por la realización de una actividad totalmente innovadora. Una temática que comúnmente resulta tediosa, incomprensible para la gran de parte de los técnicos que se forman en la UPV, resultó interesante. En consecuencia la motivación del alumno, provocó que se diera cuenta de su capacidad de diseño de una estructura, la facilidad con la que se puede comprobar su comportamiento real y lo útil que resulta la transformación mediante sus manos de la idea conceptual que tenían en su cabeza. 
2. Materialización con elementos no convencionales de una estructura singular. El uso de palos de escoba y distintos materiales de utillaje necesarios para la construcción del artefacto, muestra a los alumnos la capacidad que tienen para la concepción de maquetas las cuales, les pueden ser de gran ayuda para su formación y para su futura profesión como técnicos. En muchas ocasiones las operaciones de trabajo revelan defectos o virtudes impensables que no ofrece el diseño tradicional gráfico y que no muestra los nuevos sistemas informáticos a su disposición. Su modelado puede ayudarles a comprender, a tomar decisiones o mejorar su concepción inicial por sí mismos de forma sencilla y rápida.

3. Aplicación del trabajo colaborativo. La diversidad de alumnos integrantes del taller, sus diferentes aptitudes y diferencia de conocimiento provocan la necesidad de colaborar entre ellos para generar el elemento propuesto. El buen clima en el que se desarrolló la actividad provocó que cada uno de ellos se implicase de forma indistinta en lo que sabía hacer, proponiendo a priori sus conocimientos y lo que podía aportar para llevar a buen fin la tarea encomendada.

4. Aplicabilidad de los conocimientos adquiridos. El propio desarrollo del taller, las dudas, los problemas y las soluciones propuestas mostraron al alumno que los distintos conceptos teóricos que había interiorizado en su formación educativa tenían un sentido real. En este aspecto, no sólo se aplicaron conocimientos desarrollados dentro del ámbito universitario, si no también conceptos aprendidos durante su formación en el instituto.

Tras finalizar el taller y fuera del horario establecido, el buen ambiente de la actividad provocó por sí solo un foro de discusión de los resultados obtenidos. En este foro se comentaron distintas posibilidades, se propusieron nuevos retos y se comentaron las sensaciones surgidas tras la actividad.

\section{Conclusiones}

Las conclusiones obtenidas de la actividad pueden desarrollarse atendiendo a dos factores, por un lado personales de cada uno de los alumnos y en segundo lugar las extraídas del propio grupo de profesores coordinadores.

En cuanto a los alumnos y tras el análisis de los resultados obtenidos, las primeras conclusiones que se verificaron en cada uno de ellos es de satisfacción. Muchos de ellos se mostraban sorprendidos de la capacidad personal de trabajar en equipo, con un tiempo limitado y de forma organizada en la construcción de una estructura que a priori parecía complicada, de la que desconocían muchos de ellos su forma de trabajo y funcionamiento. Pero sobre todo lo más imponente fue su puesta en funcionamiento o en carga tras el montaje. La estructura que habían construido con sus propias manos, trabajaba tal y como se había planteado en un principio y además estaba construida con unos elementos comúnmente conocidos y a la disposición de todos por tratarse de materiales básciso. Ello mostraba que una maqueta de trabajo, no suponía un gasto adicional exagerado y que por tanto era importante su imaginación para la elección de cualquier material reciclado adecuado a sus necesidades.

(c) EY-NC-ND 2016, Universitat Politècnica de València

Congreso In-Red (2016) 
Por otro lado y desde el punto de vista del equipo de coordinación, las conclusiones extraídas fueron unánimes y se centraban básicamente en la facilidad de interacción profesor-alumno. El hecho de realizar una actividad singular, hizo que por un lado desarrollasen la capacidad creativa y práctica, pero a su vez interiorizasen el concepto estructural de esta tipología concreta, apoyándose en conocimientos que conocían pero de los que no sabían exactamente su uso concreto.

\section{Bibliografía}

CASSINELLO, P. (2006). En memoria de Heinz Hossodrof. Informes de la Construcción, vol. $58, n^{\circ} 2$

CIRIGLIANO, G. y VILLAVERDE, A. (1985). Dinámica de grupos y educación. Buenos Aires: Humanitas.

ENTWISTLE, N. (1987). La comprensión del aprendizaje en el aula. Barcelona. Paidós.

GIBB, J. R. (1989). Manual de dinámica de grupos. Buenos Aires: Humanitas.

HEYMAN, J. (1999). Teoría, historia y restauración de estructuras de fábrica: Colección de ensayos. Barcelona. Editorial Reverté.

KUBIATKO, M. y VACULOVÁ, I. (2011) Project-based learning: characteristic and the experience with application in the science subjects. Energy Education Science and Technology Part B: Social and Educational Studies Vol. 3. Issue 1, pp. 65-74.

FERNÁNDEZ, A. (2003) Nuevas metodologías docentes. Instituto de Ciencias de la Educación de la Universitat Politècnica de València.

PUERTAS, L. (1989). Estructuras espaciales desmontables y desplegables. Estudio de la obra del arquitecto Emilio Pérez Piñero. Tesis Doctoral. Madrid. Escuela Técnica Superior de Arquitectura de Madrid.

SÁNCHEZ, J. (1996). Estructuras desplegables de aspas para mallas poliédricas curvas. Tesis Doctoral. Sevilla. Universidad de Sevilla.

TORROJA, E. (1960) Razón y ser de los tipos estructurales. CSIC. Madrid.

YUS, R. (1996). Temas transversales: hacia una nueva escuela. Barcelona. Editorial Grao. 